\title{
PLANET FORMATION IN CIRCUMBINARY CONFIGURATIONS: TURBULENCE INHIBITS PLANETESIMAL ACCRETION
}

\author{
Stefano Meschiari \\ McDonald Observatory, University of Texas at Austin, Austin, TX 78712, USA; stefano@ astro.as.utexas.edu \\ Received 2012 October 8; accepted 2012 October 25; published 2012 November 19
}

\begin{abstract}
The existence of planets born in environments highly perturbed by a stellar companion represents a major challenge to the paradigm of planet formation. In numerical simulations, the presence of a close binary companion stirs up the relative velocity between planetesimals, which is fundamental in determining the balance between accretion and erosion. However, the recent discovery of circumbinary planets by Kepler establishes that planet formation in binary systems is clearly viable. We perform $N$-body simulations of planetesimals embedded in a protoplanetary disk, where planetesimal phasing is frustrated by the presence of stochastic torques, modeling the expected perturbations of turbulence driven by the magnetorotational instability. We examine perturbation amplitudes relevant to dead zones in the midplane (conducive to planet formation in single stars), and find that planetesimal accretion can be inhibited even in the outer disk (4-10 AU) far from the central binary, a location previously thought to be a plausible starting point for the formation of circumbinary planets.
\end{abstract}

Key words: planets and satellites: dynamical evolution and stability - planets and satellites: formation

Online-only material: color figures

\section{INTRODUCTION}

Among the numerous discoveries of the Kepler mission, the detection of circumbinary (CB) planets Kepler 16 through the multiple system Kepler 47 (Doyle et al. 2011; Welsh et al. 2012; Orosz et al. 2012a, 2012b) has propelled a renewed theoretical effort in explaining how such planets would be assembled in a binary environment. In the presence of a close binary companion, protoplanetary disks can become a rather hostile planetary nursery in both circumstellar and circumbinary configurations. The difficulties encountered by the standard core accretion paradigm in the binary environment are several, including truncation, mass loss and relatively fast dispersal of disks in close binaries (e.g., Duchêne 2010; Kraus et al. 2012), possible vaporization of grains in dynamically excited disks (Nelson 2000), and impaired planetesimal growth into protoplanets (Marzari \& Scholl 2000; Moriwaki \& Nakagawa 2004; Thébault et al. 2004, 2006; Scholl et al. 2007; Paardekooper et al. 2008; Thebault 2011).

The latter "planetesimal bottleneck" is a robust consequence of the interplay between the gravitational perturbations of the stellar companion (which stirs the planetesimal disk and acts to raise eccentricities) and the aerodynamic drag from a putative protoplanetary disk. Since aerodynamic drag tends to both damp planetesimal eccentricities and align planetesimal orbits in a size-dependent fashion, planetesimals of different sizes will collide on different phases, resulting in high collisional speeds which lead to destructive (rather than accreting) events. Recently, Meschiari (2012, hereafter M12) investigated planetesimal accretion in the Kepler-16 system, using $\mathrm{N}$-body simulations (coupled with drag from a static background disk) which track planetesimal collisions throughout a range of semimajor axes over $10^{5}$ years. The census of planetesimal collisions indicated that regions inside $4 \mathrm{AU}\left(\approx 20 a_{B}\right.$, where $a_{B}$ is the semimajor axis of the central binary) were dominated by destructive events, and therefore hostile to planet formation. Consequently, we posited that Kepler 16 could have plausibly assembled outside the forbidden region and subsequently migrated inward through tidal interaction with the protoplanetary disk, later stopping close to the inner edge of the disk. This scenario is supported by the evolution of planetary cores in hydrodynamical simulations (Pierens \& Nelson 2007, 2008), which also suggested that Jupiter-mass planets in CB configurations should be rare (in accordance with the observed Kepler sample). Paardekooper et al. (2012) investigated planet formation in the Kepler 16, 34, and 35 systems using a similar approach, additionally including self-consistent planetesimal formation and destruction. They reached analogous conclusions, and asserted that in situ formation was unlikely, even under the most favorable conditions.

The approach of M12, similar to previous investigations, neglected several physical ingredients for the sake of computational expediency. Indeed, fully self-consistent simulations that include the hydrodynamical response of the protoplanetary disk have shown that additional oscillations in eccentricity and longitude of pericenter of the planetesimals might be introduced from the development of bulk eccentricity and spiral perturbations in the disk. However, their magnitude might depend somewhat on the details of the hydrodynamical simulation (e.g., Paardekooper et al. 2008; Marzari et al. 2012; Müller \& Kley 2012).

In this Letter, we consider magnetohydrodynamical turbulence driven by the magnetorotational instability (MRI) as an additional source of perturbations on the planetesimal disk. MRI-driven turbulence (Balbus \& Hawley 1991) is thought to be the likely source of anomalous viscosity in protoplanetary disks (e.g., Armitage 1998), and influence how planetesimal formation (e.g., Johansen et al. 2007), planetesimal accretion (e.g., Ogihara et al. 2007; Ida et al. 2008; Nelson \& Gressel 2010), and planetary migration (e.g., Nelson \& Papaloizou 2003, 2004; Laughlin et al. 2004; Baruteau \& Lin 2010) proceed. Previous studies simulating the dynamics of planetesimals embedded in turbulent disks in single-star environments showed that in fully MRI-active disks, the velocity dispersion of the planetesimals 
is significantly raised by gravitational perturbations induced by density fluctuations. For typical disk parameters and nominal turbulence strength, km-sized planetesimals might be in a highly erosive regime. Such vigorous turbulence, however, might not be appropriate to the midplane of realistic protoplanetary disks, which is thought to be dominated by a "dead zone" with nearlaminar flow (Gammie 1996). Gressel et al. (2011, hereafter G11) presented the results of stratified, three-dimensional (3D) MHD simulations (including a substantial dead zone); planetesimals embedded in the disk midplane at $5 \mathrm{AU}$ experienced a significantly reduced excitation of their eccentricities (by a factor $\approx 10-20$ ). Therefore, they concluded dead zone represent "safe havens" for the growth of km-sized planetesimals.

Although the reduced amplitude of the random velocities excited by turbulent fluctuations is potentially conducive to planetesimal accretion in the single-star environment, the situation is more complicated in the binary environment. In the latter case, a very precise alignment between the planetesimal orbits is crucial to attaining low encounter speeds despite the substantial eccentricity of the planetesimal orbits. Random kicks diffuse planetesimals out of alignment; although aerodynamic drag would attempt to restore alignment, it will do so on a timescale that is size-dependent, once again differentially phasing planetesimals of different sizes. Finally, high-frequency radial oscillations of the planetesimal eccentricity (as would be caused by stochastic torques) would potentially lead to further orbital crossing, leading to high encounter velocities. Therefore, we anticipate that planet formation might be strongly perturbed, or even inhibited, despite the smaller turbulent amplitudes appropriate to the midplane dead zone. We couple the $N$-body code of M12 with a numerical model that approximates the stochastic torquing arising from MRI turbulence. Our analysis shows that this additional source of perturbations is potentially damaging to planet formation in the outer disk, which was previously thought to be relatively protected from destructive impacts and therefore a plausible location for core assembly (M12; Paardekooper et al. 2012).

The organization of this Letter is as follows. In Section 2, we briefly discuss our numerical model for the gravitational torques arising from MHD turbulence. In Section 3 we show the results of our simulations, and discuss them in the context of planet formation in Section 4.

\section{NUMERICAL SETUP}

In this Letter, we study planet formation by sampling planetesimal collision events between 4 and $10 \mathrm{AU}$, and compare them to velocity thresholds corresponding to destructive impacts. We consider the orbital elements of the Kepler-16 system as our prototypical configuration, and to facilitate comparison with previous investigations (M12; Paardekooper et al. 2012). We refer the reader to M12 for a description of the numerical code, initial setup and velocity thresholds.

\subsection{Turbulent Model}

We use the analytical prescription of Laughlin et al. (2004, hereafter L04) to model torques arising from density fluctuations in the disk; we include corrections to the formulation from Ogihara et al. (2007; also used in Ida et al. 2008; Baruteau $\&$ Lin 2010), and introduce small modifications that reduce its computational cost. In this model, density fluctuations are forced by a potential $\Phi$, a sum of turbulent $m$-fold modes

$$
\Phi=\gamma r^{2} \Omega^{2} \sum_{i} \xi_{i} R\left(r, r_{c, i}, \sigma_{i}\right) T\left(\tilde{t}_{i}\right) \cos \left(m \varphi-\varphi_{c, i}-\Omega_{c, i} \tilde{t}_{i}\right),
$$

where $\gamma$ sets the overall turbulent amplitude, $r_{c, i}, \varphi_{c, i}$ and $\Omega_{c, i}$ are the radial center, phase, and angular velocity of the mode (picked randomly in the disk), $m$ is sampled from a lograndom distribution between 1 and $6, \sigma_{i}=\pi r_{c, i} / 4 m$ is the radial extent of the mode, $\xi_{i}$ is sampled from a Gaussian distribution of unit variance, $\tilde{t}_{i}$ is the lifetime of the mode normalized by a timescale $\Delta t_{i}$, and $R$ and $T$ are two Gaussian-like functions centered around $r_{c, i}$ and $0.5 \Delta t_{i}$, respectively. Each mode has a limited lifetime $\Delta t_{i}$; following Baruteau \& Lin (2010), we reduce the lifetimes by a factor of 10 from the prescription of L04 in order to better match the autocorrelation timescale of 3D MHD simulations.

The actual force on the planetesimals will arise from the gravitational force of the density fluctuations induced by Equation (1). In order to proceed without the full hydrodynamical machinery, L04 used a WKB analysis to derive the following scaling for the rms torque on a planetesimal of mass $\mathcal{M}_{\mathrm{pl}}$ :

$$
\tau_{T}=C \gamma r^{4} \Omega^{2} \Sigma_{g} \frac{\mathcal{M}_{\mathrm{pl}}}{\mathcal{M}_{*}},
$$

where $\mathcal{M}_{*}$ is the mass of the central object (in our case, the total binary mass) and $\Sigma_{g}$ is the unperturbed surface density of the protoplanetary disk. While L04 also derive an approximate value for the constant $C$, the heuristic nature of the derivation (which is equivalent to a dimensional analysis, as derived by Johnson et al. 2006) suggests that a better approach would be to fit the amplitude of the perturbations $\gamma$ to the results of MHD simulations.

To compare with the results of G11, we integrated the orbits of an ensemble of 100 planetesimals, started at 5 AU with zero eccentricity and random initial phase, subject to the stochastic torques described above and no aerodynamic drag and computed the diffusion of their orbital elements. We consider several models, differing only by the turbulent amplitude $\gamma$ : model A $\left(\gamma=2.5 \times 10^{-3}\right)$ represents a fiducial turbulent amplitude appropriate to fully MRI-active disks, while models B1-B4 $\left(\gamma=2.5 \times 10^{-4}, 10^{-4}, 5 \times 10^{-5}, 2.5 \times 10^{-5}\right.$, respectively) have reduced turbulent amplitudes appropriate to the midplane of dead zones. Since $\alpha$ scales as $\gamma^{2}$ (where $\alpha$ is the usual viscosity parameter in the Shakura-Sunyaev prescription; Baruteau \& Lin 2010), the values chosen span two orders of magnitude of turbulent viscosity. Finally, for comparison with M12, we also ran simulations with no turbulence (model C).

Figure 1 shows the growth of the dispersion of the planetesimal eccentricity $\sigma(e)$ as a function of time; as expected from a random-walk process, $\sigma(e) \propto t^{1 / 2}$. Models B1 and B2 bracket well the growth of the eccentricity dispersion seen by G11 for models with dead zones; we include the reduced values of models B3 and B4 to test the robustness of our results.

We remark that it is likely that the interaction between the planetesimals and the background disk is more complicated than the model presented in this Letter; for instance, we expect the disk to be endowed with some eccentricity (e.g., Marzari et al. 2008; Marzari et al. 2012). However, for the sake of simplicity and to highlight the role of turbulence as an additional factor in dephasing planetesimal orbits, we decided to take the orthogonal approach of ignoring the self-consistent evolution of the disk. In this picture, we assume a circular disk as a maximally accretionfriendly starting point. 


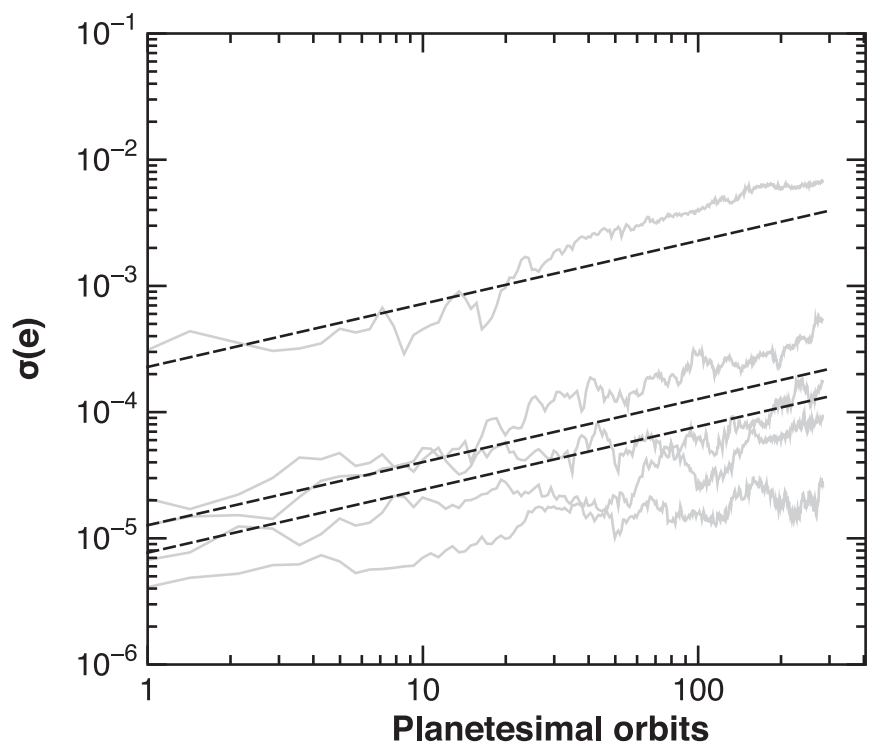

Figure 1. Sample growth plot of the eccentricity dispersion for models A and B1-B4 (gray lines, from top to bottom). The dashed lines indicate the best-fit eccentricity dispersion measured in the full MHD simulations of G11, models A, D1, and D2 (from top to bottom).

\section{SIMULATIONS}

\subsection{Diffusion of Eccentricity and Dephasing}

To visualize the effect of the turbulent fluctuation on a population of planetesimal, we first integrated the trajectories of a swarm of 10,000 planetesimals uniformly distributed between 4 and $8 \mathrm{AU}$ for four different levels of turbulence: no turbulence, model A (active-MRI disk), model B1 (nominal dead zone turbulence fitting the results of G11), and model B4 (turbulence amplitude reduced by a factor of 10 ).

Figure 2 shows the eccentricity and longitude of pericenter (ळ) of the planetesimals. Compared to the run without turbulence, both models with levels of turbulence appropriate to a dead zone add a considerable amount of noise on top of the $e$ and $\varpi$-profiles secularly imposed by the central binary. Since the orbits of neighboring planetesimals will not be as collimated, impact velocities will be raised everywhere in the disk. Therefore, we expect that the fraction of accreting impacts will be decreased even when very low levels of turbulence are present.

For the model representing a fully MRI-active disk, eccentricities are raised to very high values $\left(e \approx 5 \times 10^{-2}\right)$ and the longitude of pericenter is completely randomized; therefore, the collision velocity can be directly estimated as $\Delta v \approx e v_{\text {kep }}>400 \mathrm{~m} \mathrm{~s}^{-1}$, comfortably above any velocity threshold resulting in destructive impacts (Stewart \& Leinhardt 2009). Indeed, in this case the turbulent torques completely overwhelm the secular forcing of the central binary, resulting in high impact speeds that are consistent with the results of simulations of planetesimal dynamics in fully turbulent disks around single stars (e.g., Ida et al. 2008; Nelson \& Gressel 2010).

\subsection{Collision Statistics}

We subsequently ran full simulations with collision detection between 4 and $10 \mathrm{AU}$, for each of our models. Given the computational overhead of calculating and updating the turbulent forcing at each time step, we instead chose to integrate the trajectories of a smaller number of planetesimals $(1,000)$ concentrated in annuli centered around 4, 6, 8, and 10 AU. This
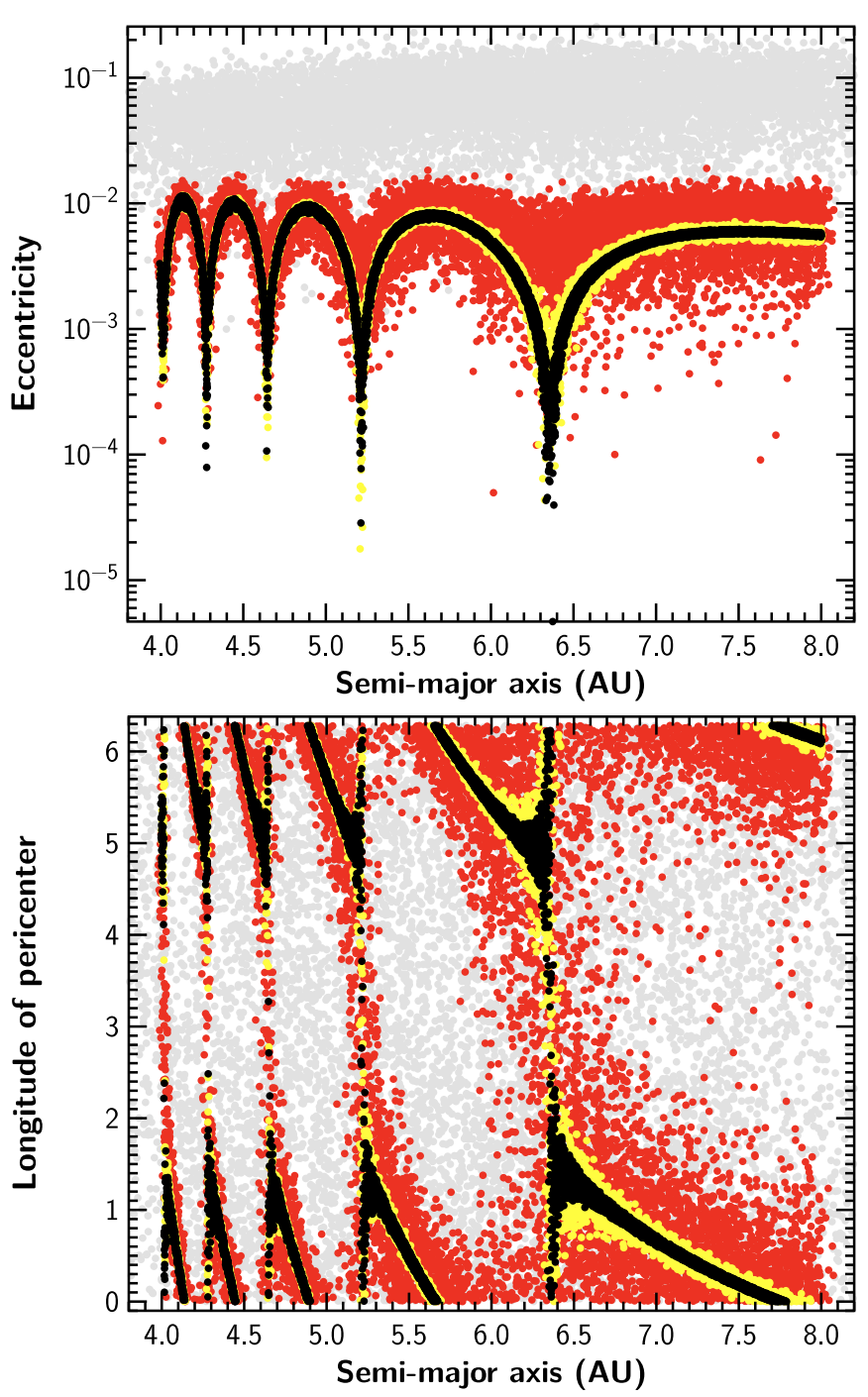

Figure 2. Eccentricity and longitude of pericenter $\varpi-\varpi_{B}$ as a function of semimajor axis for 10,000 planetesimals distributed between 4 and $8 \mathrm{AU}$, for runs with no turbulence (black dots), model A (gray dots, active-MRI disk), model B1 (red dots, dead zone), and model B4 (yellow dots, dead zone turbulence amplitude reduced by a factor of 10).

(A color version of this figure is available in the online journal.)

approach has the advantage of reducing the running time of our simulations, while simultaneously improving our collision statistics by increasing the impact rate.

Figure 3 shows the fraction of accreting impacts as a function of semimajor axis for each of our models. For the sake of completeness, we further classify impacts as "unperturbed" ( $\Delta v<v_{\text {esc }}$, where $v_{\text {esc }}$ is the escape velocity) or "perturbed" accretion $\left(v_{\text {esc }}<\Delta v<v_{\text {ero }}\right)$; for the former, the impact velocity is low enough to allow runaway growth to proceed, therefore allowing the rapid formation of oligarchs. We generously deem a radial bin as a favorable location for planet formation when the fraction of accreting impacts (unperturbed or perturbed) is larger than $50 \%$.

For models B1 and B2 (with turbulent amplitudes which best fit the results of the 3D MHD simulations of G11), we find that the percentage of accreting impacts decreases dramatically. This is consistent with the noisiness of the pericenter phasing and the high-frequency jitter around the damped value. These shortterm oscillations are not damped efficiently by the aerodynamic 


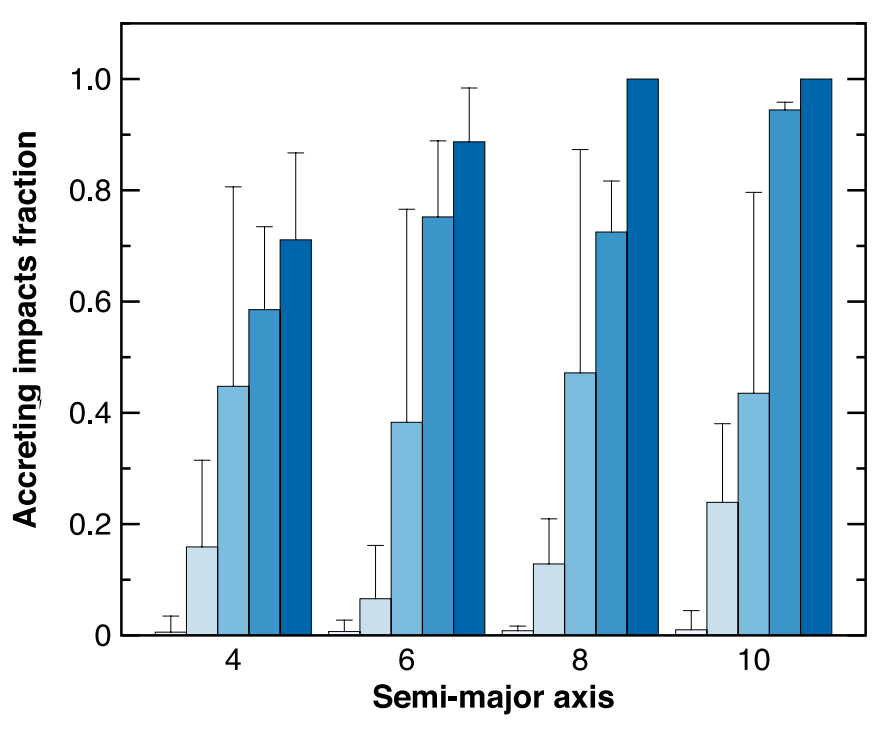

Figure 3. Fraction of accreting impacts as a function of semimajor axis (from lighter to darker: models B1-B4 and C.) Each bar represents the percentage of unperturbed impacts; the fraction of perturbed impacts is additionally represented by a solid line.

(A color version of this figure is available in the online journal.)

drag at these large distances, since the drag torque decreases steeply with distance $\left(\tau_{G} \propto a^{-3-\beta}\right.$, for planetesimals at the equilibrium forced eccentricity) while the turbulent torque in our model decreases more slowly $\left(\tau_{T} \propto a^{1-\beta}\right)$, where we take $\Sigma \propto r^{-\beta}$ ( $\beta=1.75$ for the minimum-mass solar nebula model considered in this Letter).

For models B3 and B4 (with reduced turbulent amplitudes) we find that despite the fact that substantial oscillations in eccentricity and longitude of pericenter are still induced, the resulting impact velocities have crossed the accreting threshold. The median impact speed for model B 4 is $\approx 6 \mathrm{~m} \mathrm{~s}^{-1}$, which is in the erosive regime for $1-\mathrm{km}$ planetesimals but allow accretion for $10-\mathrm{km}$ planetesimals.

Finally, in accordance with the simulations of M12, we find that planet formation can proceed undisturbed outside $4 \mathrm{AU}$ when turbulent forcing is switched off.

\section{DISCUSSION}

In this Letter we have investigated planetesimal accretion in the outer parts of a circumbinary disk (4-10 AU). Our simulations indicate that the stochastic forcing of turbulent perturbations will frustrate planetesimal phasing and raise eccentricities, inhibiting planet formation even quite far from the central binary. This result is robust for levels of turbulence that match those observed in realistic MHD simulations. In runs modeling disks fully invaded by MRI turbulence, planetesimal phasing is completely destroyed and planetesimals will collide at speeds that are much higher than fiducial erosive velocities. We then ran simulations fitting the turbulent amplitude corresponding to the stratified disks endowed with a large dead zone obtained in G11, resulting in a reduction by a factor $\approx 10-20$ in the turbulent torque. While the reduced stochastic perturbations would be sufficiently small to allow accretion in a single-star environment, in our circumbinary configuration the fraction of impacts resulting in accreting events was greatly reduced $(\lesssim 1 \%-30 \%$ between 4 and $10 \mathrm{AU}$ for models B1 and B2, respectively) due to the increased eccentricity jitter and dephasing. Therefore, it is possible to inhibit planet formation by two different mechanisms: by high eccentricities, differential phasing, and perturbations caused by the self-consistent reaction of the gas disk at small radii, and by stochastic turbulent torques at large radii. This is problematic, since if that picture is accurate, it is not feasible to form the observed planetary census at any realistic distance from the binary. The circumbinary environment is less robust than single stars with regard to dynamical perturbations induced by the disk (regardless of their origins); a further reduced level of turbulence may be required to form planets at all. Indeed, we found that further reduction of the turbulent amplitude by a factor of 2-5 was necessary to make planet formation viable again in the outer disk.

Several uncertainties in our model may still provide some room for allowing planet formation despite the perturbing effects of turbulence. Our analytic prescription, while attempting to match MHD simulations, only provides a "Oth" order description of gravitational perturbations induced by torques. For instance, we remark that we tuned the amplitude of turbulent torques based on the evolution of swarm of planetesimals in a single MHD simulation, and rescaled the turbulent amplitude at each radial location according to the numerical prescription of Section 2; however, the output of these models will depend on the assumed magnetic field, the ionization level, and possibly the resolution of the shearing box. The detailed radial dependence and even extent of the dead zone remain uncertain and depend on the assumed model (e.g., Matsumura \& Pudritz 2005; Terquem 2008; Flaig et al. 2012; if the dead zone extends to only a few AU, then fully active turbulence may still play a role in the outer disk and even shut off planetesimal accretion completely). Effectively, using the torque scaling of Equation (2) implicitly assumes that the turbulent torque is well described by the fully turbulent model of L04, attenuated by a constant factor at each radial location. Only self-consistent (including the time-dependent potential of the binary), computationally expensive global simulations could, in principle, inform the model presented in this Letter. However, since we have considered various values of $\gamma$ for each independent annulus, we are reasonably covering a number of possibilities for the radial dependence of the turbulence amplitude. Our results at a given radius should only be considered suggestive of further challenges to planet formation.

Finally, we remark that similar to M12, we have chosen to study planetesimal growth through mutual collisions in the 1-10 km planetesimal regime. A more sophisticated approach following the evolution of the size distribution of the planetesimals (adding a significant computational overhead) may be warranted; in the latter approach, coevolution with a disk of small dust might make accretion possible closer to the binary (assuming a high dust accretion efficiency; Paardekooper et al. 2012). Even in this setup, some amount of migration is needed to bring the fully formed core (or embryos) to the current location. The assumption of a static gas background is not realistic, especially in the inner disk; in that case, self-consistent gas dynamics will act to further increase impact velocities in the inner disk (Paardekooper et al. 2008). We plan to incorporate the full hydrodynamical evolution of the gas disk in a follow-up paper using the SPH module of our code.

Our simulations indicate that planetesimal accretion in the $1-10 \mathrm{~km}$ range will be inhibited everywhere in the disk, and could only proceed if the strength of the turbulent torques is reduced from our fiducial value, or the initial planetesimal population is comprised of bigger objects. The latter scenario 
is particularly appealing, as it could allow planet formation to proceed in other highly dynamically disturbed environments (e.g., Thebault 2011). Indeed, our fiducial model for dead zone turbulence becomes accretion-friendly for a planetesimal size spectrum spanning 10-100 km size. Recent simulations of planetesimal formation in weakly turbulent disks show that massive bound clumps are formed rapidly from meter-sized boulders within pressure bumps, for typical nebula parameters (e.g., Johansen et al. 2007, 2011). Such bound clumps will likely result in planetesimals with sizes comparable to at least a substantial fraction of the dwarf planet Ceres. We suggest that a primordial population of large planetesimals might be crucial to proceed with planet formation in highly perturbed environments. However, further simulations will be required to assess whether the formation of large clumps is robust to the dynamical perturbations of a binary companion.

S.M. acknowledges insightful discussions with G. Laughlin and N. Haghighipour, a useful critique from the anonymous referee, as well as support from the W. J. McDonald Postdoctoral Fellowship.

\section{REFERENCES}

Armitage, P. J. 1998, ApJ, 501, L189

Balbus, S. A., \& Hawley, J. F. 1991, ApJ, 376, 214

Baruteau, C., \& Lin, D. N. C. 2010, ApJ, 709, 759

Doyle, L. R., Carter, J. A., Fabrycky, D. C., et al. 2011, Science, 333, 1602

Duchêne, G. 2010, ApJ, 709, L114

Flaig, M., Ruoff, P., Kley, W., \& Kissmann, R. 2012, MNRAS, 420, 2419
Gammie, C. F. 1996, ApJ, 457, 355

Gressel, O., Nelson, R. P., \& Turner, N. J. 2011, MNRAS, 415, 3291

Ida, S., Guillot, T., \& Morbidelli, A. 2008, ApJ, 686, 1292

Johansen, A., Klahr, H., \& Henning, T. 2011, A\&A, 529, A62

Johansen, A., Oishi, J. S., Mac Low, M.-M., et al. 2007, Nature, 448, 1022

Johnson, E. T., Goodman, J., \& Menou, K. 2006, ApJ, 647, 1413

Kraus, A. L., Ireland, M. J., Hillenbrand, L. A., \& Martinache, F. 2012, ApJ, 745,19

Laughlin, G., Steinacker, A., \& Adams, F. C. 2004, ApJ, 608, 489

Matsumura, S., \& Pudritz, R. E. 2005, ApJ, 618, L137

Marzari, F., Baruteau, C., Scholl, H., \& Thebault, P. 2012, A\&A, 539, A98

Marzari, F., \& Scholl, H. 2000, ApJ, 543, 328

Marzari, F., Thébault, P., \& Scholl, H. 2008, ApJ, 681, 1599

Meschiari, S. 2012, ApJ, 752, 71

Moriwaki, K., \& Nakagawa, Y. 2004, ApJ, 609, 1065

Müller, T. W. A., \& Kley, W. 2012, A\&A, 539, A18

Nelson, A. F. 2000, ApJ, 537, L65

Nelson, R. P., \& Gressel, O. 2010, MNRAS, 409, 639

Nelson, R. P., \& Papaloizou, J. C. B. 2003, MNRAS, 339, 993

Nelson, R. P., \& Papaloizou, J. C. B. 2004, MNRAS, 350, 849

Ogihara, M., Ida, S., \& Morbidelli, A. 2007, Icarus, 188, 522

Orosz, J. A., Welsh, W. F., Carter, J. A., et al. 2012a, ApJ, 758, 87

Orosz, J. A., Welsh, W. F., Carter, J. A., et al. 2012b, Science, 337, 1511

Paardekooper, S.-J., Leinhardt, Z. M., Thebault, P., \& Baruteau, C. 2012, ApJ, 754, L16

Paardekooper, S.-J., Thébault, P., \& Mellema, G. 2008, MNRAS, 386, 973

Pierens, A., \& Nelson, R. P. 2007, A\&A, 472, 993

Pierens, A., \& Nelson, R. P. 2008, A\&A, 483, 633

Scholl, H., Marzari, F., \& Thébault, P. 2007, MNRAS, 380, 1119

Stewart, S. T., \& Leinhardt, Z. M. 2009, ApJ, 691, L133

Terquem, C. E. J. M. L. J. 2008, ApJ, 689, 532

Thebault, P. 2011, Celest. Mech. Dyn. Astron., 111, 29

Thébault, P., Marzari, F., \& Scholl, H. 2006, Icarus, 183, 193

Thébault, P., Marzari, F., Scholl, H., Turrini, D., \& Barbieri, M. 2004, A\&A, 427, 1097

Welsh, W. F., Orosz, J. A., Carter, J. A., et al. 2012, Nature, 481, 475 